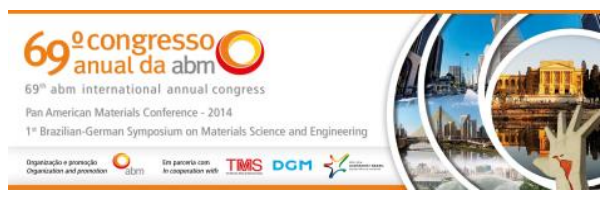

Tema: Gestão de Manutenção

\title{
COMANDO REMOTO DE ACIONAMENTO, EXTRAÇÃO E INSERÇÃO DE DISJUNTORES DE ALTA TENSÃO*
}

\author{
Elvis Candeias Silva ${ }^{1}$ \\ Jucemar Carvalho de Freitas ${ }^{2}$ \\ Helio Martins Junior ${ }^{3}$
}

\section{Resumo}

Introdução: A automatização dos procedimentos de desligamento, religamento e de extração remota de disjuntores de alta tensão consiste em um sistema que utiliza as tecnologias disponíveis na atualidade para trabalhar em prol da saúde e segurança dos trabalhadores. O conhecimento e utilização de Controlador Lógico Programável - CLP, Interfaces Homem Máquina - IHM foram considerados fundamentais para este projeto. Estes recursos alinhados às normas técnicas vigentes, e a adoção de dispositivos de proteções entre outros, possibilitou a elaboração deste projeto. Objetivo: Aumentar a segurança do eletricista ao realizar manobras com disjuntores de alta tensão. Método: Desenvolvido um sistema automatizado para desligamento, religamento, extração e inserção de disjuntores de alta tensão remotamente, utilizando um motor elétrico no painel do disjuntor, substituindo o que antes era feito por um profissional habilitado na área elétrica. Resultado: A implantação deste sistema permite realizar os procedimentos no disjuntor em local seguro, chamado zona livre, distante do raio de ação de qualquer energia proveniente de um arco elétrico. Conclusão: O projeto permitiu a remoção do risco para o eletricista durante a operação do disjuntor, deixando-o livre de qualquer possível contato com a energia proveniente de uma falha no disjuntor.

Palavras-chave: Disjuntor; Comando remoto; IHM; Segurança.

\section{REMOTE DRIVE, EXTRACTION AND INSERTION OF HIGH VOLTAGE CIRCUIT BREAKERS}

\begin{abstract}
Introduction : The automation of procedures for shutdown, restart remotely and extraction of high voltage circuit breakers consist of a system which uses the technologies available today to work for the health and safety of workers. The knowledge and use of Programmable Logic Controller - PLC, Human Machine Interface - HMI were the key to this project. These resources aligned to current technical standards, and the adoption of protections and other devices enabled the development of this project. Objective: Increase the safety and health of the electrician during high voltage circuit breakers operations. Method: Developed an automated system for shutdowns, restart, extraction and insertion of high voltage circuit breakers remotely using an electric motor at circuit breaker cabinet, replacing what was previously done by a qualified electrical professional. Results: The implementation of this system allows the worker to perform procedures at the circuit breaker in a safe place, called free zone, away from the radius of action of any energy from an electrical arc. Conclusion: The project enabled the removal of the electrician risk during breaker operation, being free from any possible contact with energy from a breaker failure.

Keywords: Breaker; Remote control; HMI; Safety.

1 Engenheiro Eletricista, Supervisor de Controle de Processo, Setor de produção de placas, ArcelorMittal Tubarão, Serra, ES, Brasil.

2 Bacharel em Sistema de Informação, Supervisor de Controle de Processo, Setor de produção de placas, ArcelorMittal Tubarão, Serra, ES, Brasil.

3 Técnico em Automação Industrial, Técnico de inspeção elétrica, Setor de produção de placas, ArcelorMittal Tubarão, Serra, ES, Brasil.
\end{abstract}

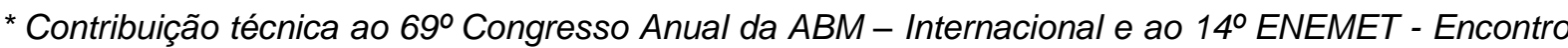
Nacional de Estudantes de Engenharia Metalúrgica, de Materiais e de Minas, 21 a 25 de julho de 2014, São Paulo, SP, Brasil.
} 


\section{INTRODUÇÃO}

Um disjuntor é um dispositivo eletromecânico, que é utilizado como meio de interromper uma corrente elétrica em um circuito através de aberturas de contatos mecânicos utilizados como caminho da corrente elétrica [1]. Esta interrupção pode ser involuntária ou proposital, dependendo da situação atual que o circuito esteja vivendo. Em uma atuação involuntária o disjuntor trabalha quando ocorre sobrecarga e/ou curto-circuito na linha onde este estiver inserido, trabalhando conforme seus parâmetros de sobrecarga, seletividade, curva entre outros [1]. Em atuações propositais o disjuntor trabalha como uma seccionadora [2], abrindo os contatos e impedindo a passagem da corrente elétrica. Essa manobra de seccionamento é feito pelo homem, sendo este necessário atuar diretamente no equipamento. A utilização do disjuntor como dispositivo de manobra traz grandes riscos ao indivíduo que ali esteja fazendo uso deste equipamento, visto que qualquer falha em sua sequência de funcionamento ou um defeito interno pode resultar em um acidente, onde as consequências são incalculáveis.

Conforme estabelecido pela Norma Regulamentadora 10 - NR10 Segurança em Instalações e Serviços em Eletricidade, no item 10.2 Medidas de controle, é obrigatório a adoção de medidas de controle que venham a proteger o trabalhador quanto ao risco de lesões durante atividades que por ventura possam causar lesões em momentos de falhas e possíveis acidentes [3].

Em meio a tanta tecnologia disponível na atualidade, a utilização da eletroeletrônica [4] pode além do conforto, produzir ao indivíduo segurança.

Este estudo teve como objetivo automatizar os comandos de ligar, desligar, extrair e inserir disjuntores de alta tensão da subestação da planta de Lingotamento Contínuo 2 da companhia siderúrgica ArcelorMittal Tubarão, aplicando a tecnologia atual na construção de máquinas e equipamentos que venham a substituir o trabalhador nas atividades de elevado grau de risco.

\section{MATERIAIS E MÉTODOS}

Estudo descritivo de caráter qualitativo realizado na subestação de distribuição elétrica do Lingotamento Contínuo 2 da CIA Siderúrgica ArcelorMittal Tubarão, no município de Serra - ES, no período de fevereiro de 2011 a julho de 2012.

Foram observados os disjuntores pertencentes à família VB1 13.8-750-3 do fabricante GE (Figura 1). Os comandos de ligar e desligar por padrão são da forma manual, bem como a extração e inserção. Foi inserido o controlador PLC5 da Rockwell Automation, com cartão de saída digital isolada com contato a relé, para fazer acionamento remoto do comando de desliga e liga comandada pela IHM Painelview da Rockwell Automation.

A lógica de controle foi criada na ferramenta de edição RsLogix5, comunicação serial RS-232, drive de comunicação Rslinx classic. Já para a criação das telas da IHM foi utilizado o software de edição Factory talk edition, sendo este carregado na IHM através de comunicação ethernet com a utilização de cabo cross-over.

Primeiramente foram criados os comandos remotos liga e desliga. A botoeira de comando manual de liga foi emparelhada por uma saída digital do PLC5, quando acionada fecha o circuito de comando ligando o disjuntor. Da mesma forma foi com o comando desliga, neste caso desligando o disjuntor.

\footnotetext{
* Contribuição técnica ao 69ำ Congresso Anual da ABM - Internacional e ao 14ํㅡㄹ ENEMET - Encontro Nacional de Estudantes de Engenharia Metalúrgica, de Materiais e de Minas, 21 a 25 de julho de 2014, São Paulo, SP, Brasil.
} 

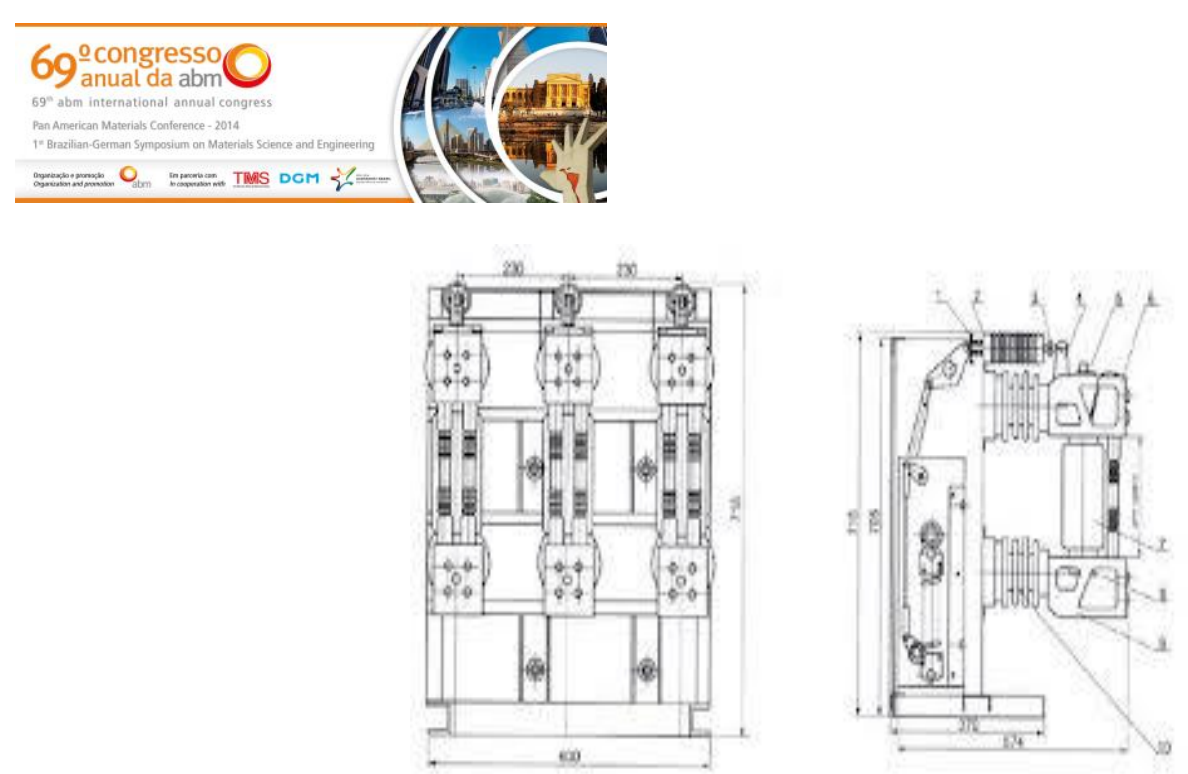

Figura 1. Disjuntor VB1 13.8-750-3

Os trabalhadores desta subestação atuaram dentro das zonas de acordo com o nível de tensão do circuito. Estas têm uma classificação de exposição aos efeitos da energia incidente deste painel. As classificações das Zonas dependerão do nível de tensão e da distância do trabalhador ao ponto da instalação energizada (Figura 2).

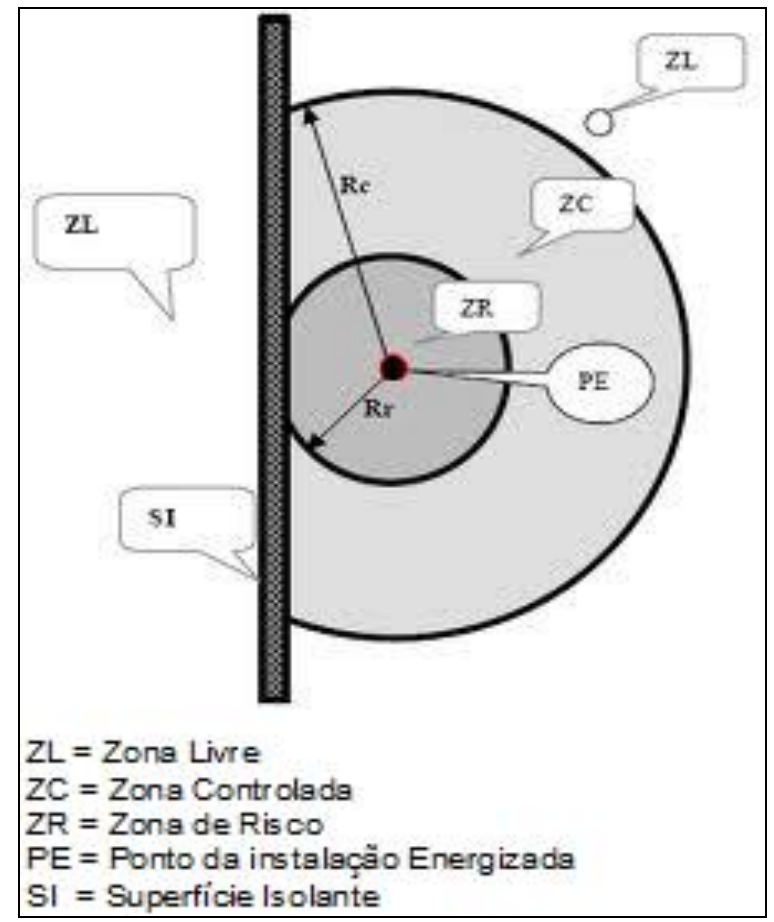

Figura 2. Classificação das zonas.

Segunda etapa do estudo foi a criação de um dispositivo de extração e inserção (Figura 3). Este dispositivo foi criado usando um motor elétrico $127 \mathrm{Vac}$, acoplado ao fuso sem fim e suporte para fixação no painel do disjuntor. Foram realizados testes manuais para se calcular um tempo satisfatório para se manter habilitado o comando do processo de extração e inserção, visto que o encerramento do acionamento do motor se dá pela atuação do sensor que fica em série com a fase e atua abrindo o circuito quando ocorre o travamento do eixo do rotor, indicando que seu curso chegou ao final.

Efetuado estudo no funcionamento eletromecânico do dispositivo, verificando possibilidades de intervenção no mesmo de forma que fosse possível uma atuação à

\footnotetext{
* Contribuição técnica ao $69^{\circ}$ Congresso Anual da ABM - Internacional e ao 14ํㅡㄹ ENEMET - Encontro Nacional de Estudantes de Engenharia Metalúrgica, de Materiais e de Minas, 21 a 25 de julho de 2014, São Paulo, SP, Brasil.
} 


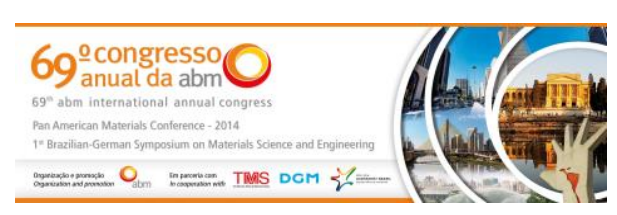

distância. Dessa forma a configuração deste dispositivo foi alterada para a realidade do projeto.

Criado um novo ponto na lógica do PLC5, acionando uma saída digital para comandar o dispositivo de extração e inserção. Agora remotamente. A saída digital está alimentando um contator que quando acionado, liga ou desliga o motor. Esse motor acoplado a manivela de extração/ inserção, substitui o acionamento manual, antes feito pelo eletricista. O comando de extração e inserção foi implementado na IHM, que envia uma solicitação ao PLC e este verifica todos intertravamentos de segurança e pede confirmação da atuação.

A IHM foi implantada na zona livre, dispensando o uso das vestimentas de segurança obrigatória.

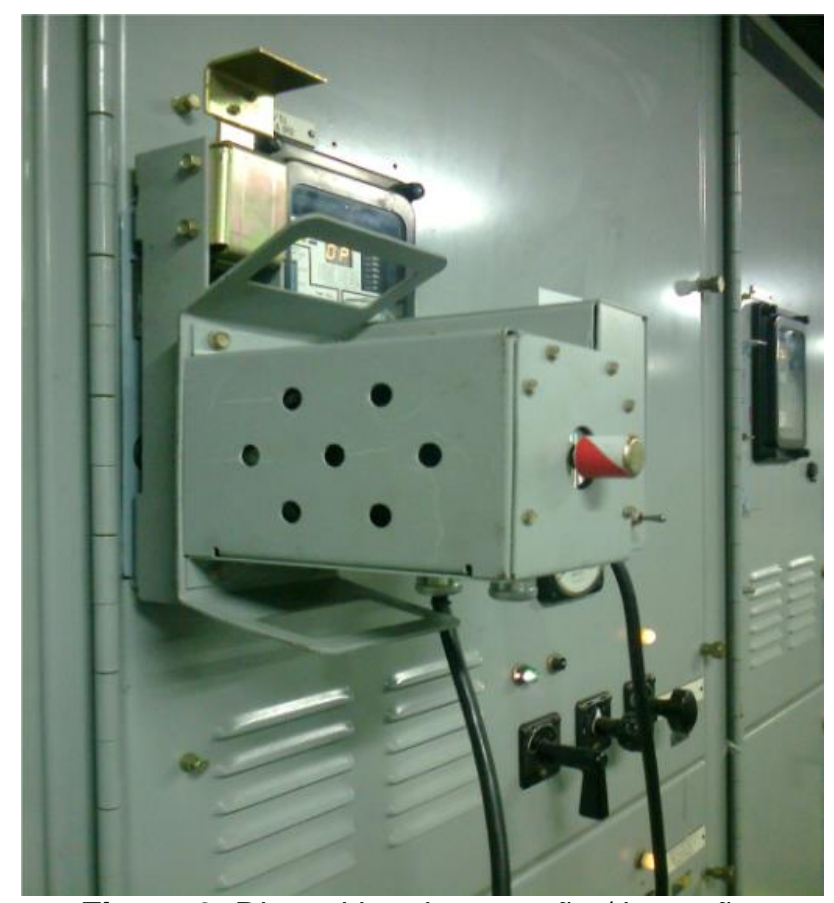

Figura 3. Dispositivo de extração / inserção.

\section{RESULTADOS E DISCUSSÃO}

Com a implantação deste sistema foi percebido pelos trabalhadores da planta uma maior segurança durante a realização das suas atividades. Fato de fácil percepção quanto ao ganho é a identificação do equipamento a ser desligado e manobrado, onde a própria IHM em sua tela (Figura 4) oferece um layout de fácil interpretação e impõe a necessidade de confirmação da informação através de telas de confirmação de manobra diminuindo significativamente a possibilidade de o eletricista atuar no equipamento errado em virtude da sua semelhança e proximidade.

\footnotetext{
* Contribuição técnica ao $69^{\circ}$ Congresso Anual da ABM - Internacional e ao 14ํㅡㄹ ENEMET - Encontro Nacional de Estudantes de Engenharia Metalúrgica, de Materiais e de Minas, 21 a 25 de julho de 2014, São Paulo, SP, Brasil.
} 

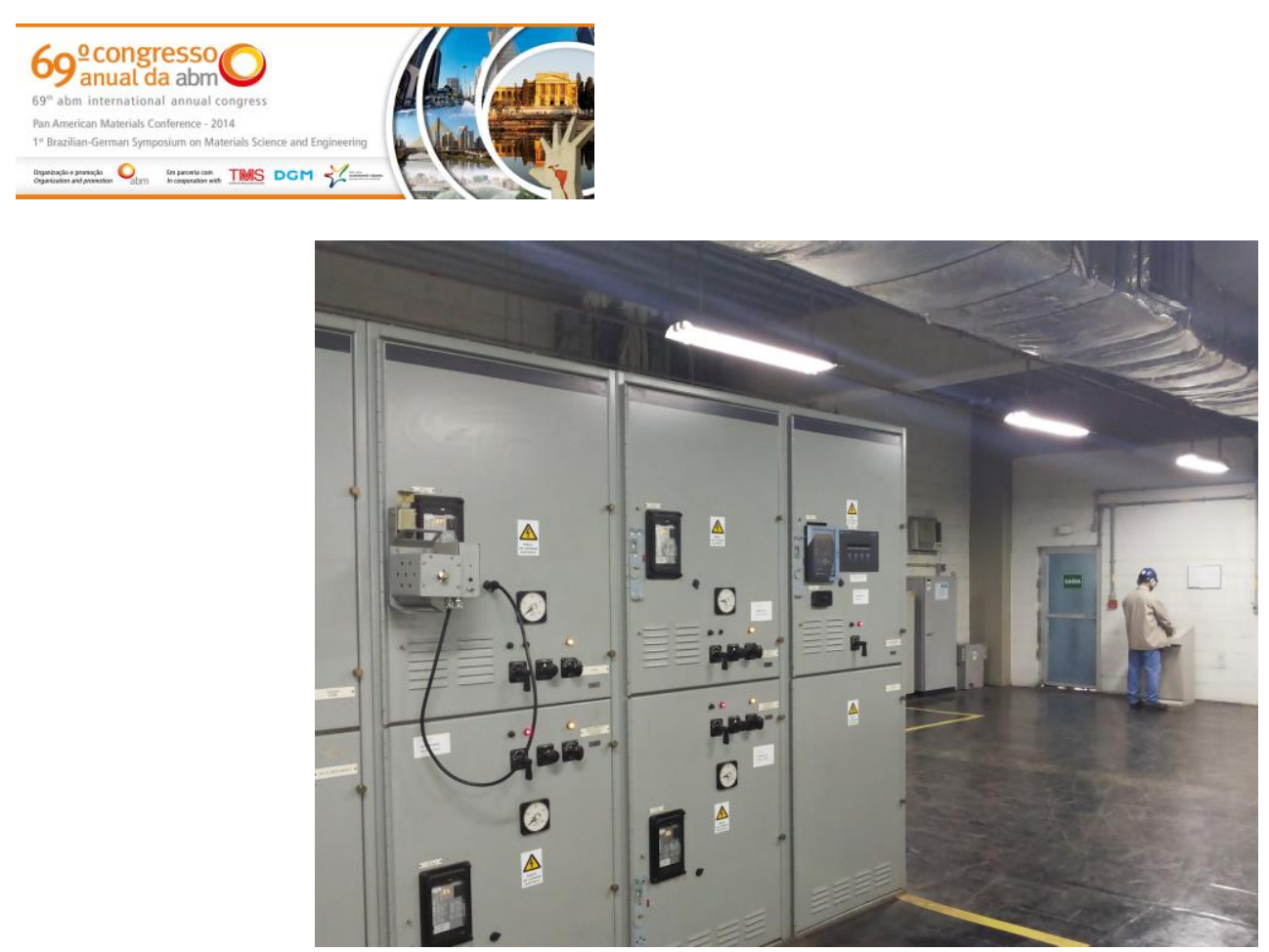

Figura 6. Extração remota do disjuntor.

De acordo com Costa [5], existe um elevado retorno ao se investir na redução dos acidentes elétricos, pois evita-se resultados indesejados como afastamento do profissional, tratamentos prolongados causados por queimaduras ou traumatismos, problemas familiares, emocionais e sociais.

Costa [5], apresenta as estatísticas de acidentes de 2004 com 129 acidentes fatais relacionadas com eletricidade, sendo o principal motivo o arco elétrico.

Segundo a ABRACOPEL [6] (Associação Brasileira de Conscientização para os Perigos da Eletricidade), em 2011 foram 298 mortes causadas pela eletricidade. Isso nos mostra que houve um aumento significativo no número de acidentes com eletricidade. Por outro lado observa-se uma incoerência de resultados, pois através da Portaria 598 do MTE de 07/12/2004 foram instituídos prazos para implantação da NR-10, o que se esperava uma diminuição no número de acidentes, principalmente os fatais.

As definições de distância de segurança zona de risco e controlada, pela NR-10, podem ter grande resultados para o risco de choque elétrico por contato direto, mas não na sua totalidade, segurança adequada para exposição ao risco do arco elétrico. Ralph Lee [7] desenvolveu equações gerais utilizáveis para todos os níveis de tensão para o cálculo da energia incidente e da distância de segurança do operador, porém sem utilizar a corrente de arco. O IEEE [8] e NFPA 70E [9] aperfeiçoaram as equações através de testes introduzindo o cálculo da corrente de arco e formularam as equações que são atualmente utilizadas para níveis de até $15 \mathrm{kV}$.

Para Costa ${ }^{[5]}$, existem várias tecnologias modernas de controle a exposição ao arco elétrico. Umas delas vêm ao encontro deste trabalho, que é o uso do controle remoto para comando e extração/inserção de disjuntores e contatores. Costa [5] afirma que o arco elétrico é um fenômeno que introduz grandes riscos para as pessoas que intervêm nos sistemas elétricos. Por esse motivo os engenheiros devem conhecer os recursos modernos para seu controle para projetar sistemas elétricos seguros.

No caso o projeto foi desenvolvido numa subestação em operação, o que permite abrir o leque para outros projetos já existentes. Segundo a norma IEEE C37. 59 (IEEE Standard Requirements for Conversion of Power Switchgear Equipment) [10], na sua edição de 2002, a opção existente para a conversão de equipamentos de manobra de potência seria a Reforma (Retrofit)/ Modernização (Upgrading) [11].

* Contribuição técnica ao $69^{\circ}$ Congresso Anual da ABM - Internacional e ao 14ํㅡㄹ ENEMET - Encontro Nacional de Estudantes de Engenharia Metalúrgica, de Materiais e de Minas, 21 a 25 de julho de 2014, São Paulo, SP, Brasil. 


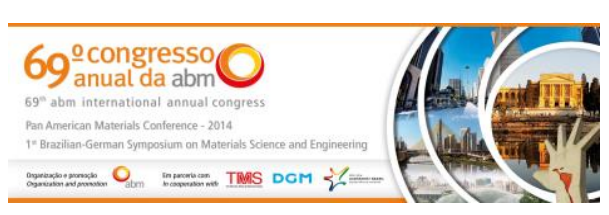

De acordo com Costa [12], nem sempre é viável a total substituição de um equipamento. Podem existir demandas de produção, limitações de espaço, restrições operacionais, pouca disponibilidade de tempo e até falta de verbas. Mas o fato concreto é que um equipamento inadequado ou métodos obsoletos podem provocar acidentes que resultem em muitos transtornos e em custos consideráveis, os quais, muitas vezes, acabam por ser pagos pelo ser humano.

Com a modernização do sistema de manobra do disjuntor VB1 13.8-750-3 na subestação da planta de lingotamento contínuo 2, continuamos atendendo todas as recomendações da NR-10, bem como as distâncias seguras definidas por Ralph Lee [7] e melhoradas pelo IEEE [8] e NFPA 70E [9] e eliminamos os riscos do arco elétrico para as pessoas que trabalham no procedimento de extração e inserção destes disjuntores.

Os resultados desse estudo sugerem que com o processo de modernização (Upgrading) dos conjuntos de manobra comandando remotamente a extração/inserção de disjuntores e aplicação desta em novos projetos, os trabalhadores terão mais segurança em suas atividades, maior confiabilidade nos procedimentos, maior produtividade, pois não perderão tempo com execuções manuais e maior conforto por não necessitar usar vestimentas anti-chama. Além do mais, as estatísticas de acidentes irão diminuir, trazendo um grande retorno para as empresas.

\section{CONCLUSÃO}

A modernização dos conjuntos de manobra utilizando comando remoto de desligamento/religamento e a extração/inserção de disjuntores de alta tensão aumenta significativamente a segurança dos trabalhadores que atuam diretamente com este equipamento, visto que qualquer problema que venha a ocorrer durante as manobras não atingirá o empregado por este estar na zona livre fora do raio de ação de qualquer material ou energia que venha romper as barreiras do painel e se projetar para o meio externo.

\section{Agradecimentos}

À equipe de engenharia da ArcelorMittal Tubarão que colaborou na especificação do motor elétrico, à equipe de controle e processo com a participação nos testes e à equipe de execução pela qualidade da montagem dos componentes.

\section{REFERÊNCIAS}

1 Mamede Filho J. Instalações elétricas industriais. 8ª ed. Rio de Janeiro: LTC; 2010. Dispositivos de manobra; p. 95-96.

2 Mamede Filho J. Instalações elétricas industriais. 8ª ed. Rio de Janeiro: LTC; 2010. Dispositivos de manobra; p. 96-97

3 Ministério do Trabalho e Emprego. NR10: Segurança em Instalações e Serviços em Eletricidade. Brasília; 2004.

4 Henriques RVB. Programação e simulação de Robôs. In: Romano VF. Robótica Insdustrial: aplicação na insdústria de manufatura e de processos. Rio de Janeiro: Editora Edgard Blücher Ltda; 2002. p. 110-117.

5 Costa PF. Mini curso - Arco eléctrico. In: Anais do 6o Encontro Sênior de Engenharia Elétrica; 2010; Belo Horizonte, Brasil. 2010. p.1-70.

\footnotetext{
* Contribuição técnica ao $69^{\circ}$ Congresso Anual da ABM - Internacional e ao 14ํㅡㄹ ENEMET - Encontro Nacional de Estudantes de Engenharia Metalúrgica, de Materiais e de Minas, 21 a 25 de julho de 2014, São Paulo, SP, Brasil.
} 
6 Associação Brasileira de Conscientização para os Perigos da Eletricidade ABRACOPEL: estatísticas acidentes de origem elétrica em 2011. 2011 [acesso em 22 out. 2012]. Disponível em:http://www.abracopel.org.br/wpcontent/uploads/2012/02/estat\%C3\%ADstica.pdf.

7 Lee $\mathrm{RH}$. The other electrical Hazard: Electric arc blast burns. [acesso em 18 out. 2012]. Disponível em: http://www.electrician2.com/html/elec_haz.pdf.

8 IEEE - Institute of Electrical and Electronics Engineers. Disponível em: http://www.ieee.org.br/

9 National Fire Protection Association. NFPA-70E - Standard for electrical safety in the workplace. [acesso em 18 out. 2012]. Disponível em: http://www.nfpa.org.

10 Institute of Electrical and Electronics Engineers. IEEE C37. 59 - Standard Requirements for Conversion of Power Switchgear Equipment. São Paulo; 2002.

11 Institute of Electrical and Electronics Engineers. IEEE C37. 59 - Standard Requirements for Conversion of Power Switchgear Equipment Retrofiting. São Paulo; 2002.

12 Costa LF. Soluções para a conversão de disjuntores e de conjuntos de manobra e controle de média e baixa tensão. Atitude editorial. 2009 [acesso em 19 out. 2012]; 42 ed.. Disponível em: http://www.osetoreletrico.com.br/web/a-revista/edicoes/122solucoes-para-a-conversao-de-disjuntores-e-de-conjuntos-de-manobra-e-controle-demedia-e-baixa-tensoes.html .

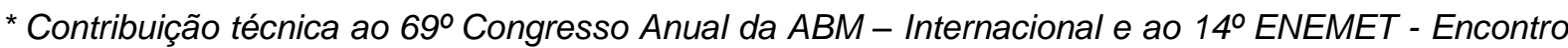
Nacional de Estudantes de Engenharia Metalúrgica, de Materiais e de Minas, 21 a 25 de julho de 2014, São Paulo, SP, Brasil. 